\title{
Subcutaneous dextrose for rehydration of elderly patients - an evidence-based review
}

\author{
Tari Turner*1 and Anne-Marie Cassano ${ }^{2}$
}

Address: ${ }^{1}$ Centre for Clinical Effectiveness, Monash Institute of Health Services Research, Monash Medical Centre, Clayton, Victoria, Australia and 2Rehabilitation and Aged Care Services, Kingston Centre, Cheltenham, Victoria, Australia

Email: Tari Turner* - tari.turner@med.monash.edu.au; Anne-Marie Cassano - Annemarie.cassano@southernhealth.org.au

* Corresponding author

Published: 15 April 2004

BMC Geriatrics 2004, 4:2
Received: 02 December 2003

Accepted: 15 April 2004

This article is available from: http://www.biomedcentral.com/l47/-23I8/4/2

(C) 2004 Turner and Cassano; licensee BioMed Central Ltd. This is an Open Access article: verbatim copying and redistribution of this article are permitted in all media for any purpose, provided this notice is preserved along with the article's original URL.

\begin{abstract}
Background: In the Rehabilitation and Aged Care Services Program at Southern Health in Victoria, saline hypodermoclysis is a relatively common method of rehydration. However, there were questions about the safety and effectiveness of subcutaneous infusion of other fluids and, in particular, dextrose solutions. This review aimed to assess the safety and effectiveness of rehydration of elderly patients with subcutaneous $5 \%$ dextrose solutions compared with intravenous $5 \%$ dextrose solutions.
\end{abstract}

Methods: We searched the Cochrane Library, Medline, IDIS, CINAHL, Current Contents, Premedline, Australasian Medical Index, the Joanna Briggs Institute, the US National Guideline Clearinghouse and bibliographies of retrieved articles. Searching was undertaken in July 2003. Studies selected were primary studies (or systematic reviews of primary studies) providing evidence as to the effectiveness and safety of subcutaneous infusion of dextrose solutions for rehydration of elderly patients. We included articles published in English in the last 10 years. Data were extracted by a single researcher.

Results: From our search we identified I 5 potentially relevant articles. We obtained the full text of these articles to determine their relevance. After application of the inclusion criteria, four articles remained for appraisal including one systematic review, two randomised controlled trials and one cohort study.

Conclusion: The four studies appraised all provide evidence that appropriate volumes of subcutaneous dextrose infusions (in the form of half-normal saline-glucose $5 \%, 40 \mathrm{~g} / \mathrm{L}$ dextrose and $30 \mathrm{mmol} / \mathrm{L} \mathrm{NaCl}$, or $5 \%$ dextrose solution and $4 \mathrm{~g} / \mathrm{L} \mathrm{NaCl}$, or two-thirds $5 \%$ glucose and one-third normal saline) can be used effectively for the treatment of dehydration, with similar rates of adverse effects to intravenous infusion. The evidence in this area is limited, and larger randomised controlled trials using validated outcome measures would be useful to confirm these results.

\section{Background}

Hypodermoclysis, or subcutaneous fluid infusion, was widely used to treat dehydration in the first half of last century. However after reports of severe adverse reactions in the 1950s [1], predominantly resulting from infusion of hypertonic solutions, hypodermoclysis fell into disrepute and then almost entirely out of use. 
Table I: Data Sources (Search date)

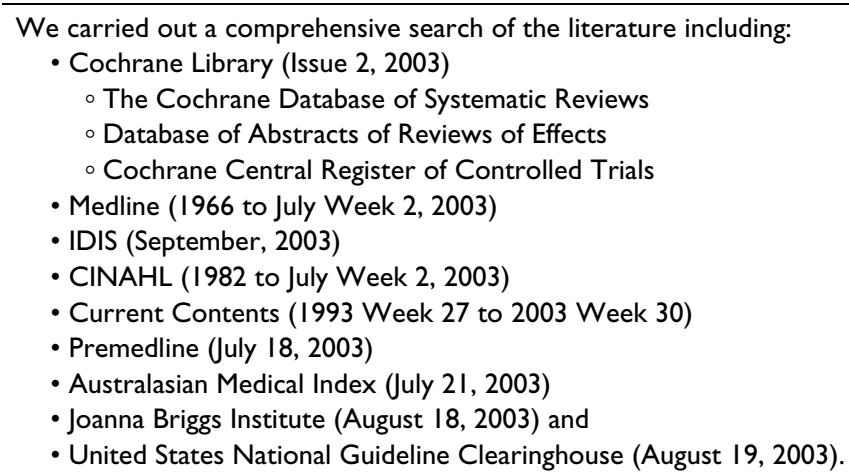

A physician from Rehabilitation and Aged Care Services at Southern Health contacted the Centre for Clinical Effectiveness to ask that we investigate the safety and effectiveness of subcutaneous dextrose solutions in dehydrated patients aged over 65 years. In the Rehabilitation and Aged Care Services Program saline hypodermoclysis is a relatively common method of rehydration. However, there were questions about the safety and effectiveness of subcutaneous infusion of other fluids and, in particular, dextrose solutions.

In consultation with the physician we undertook a comprehensive review to provide evidence to answer two questions: "In elderly patients, is rehydration with subcutaneous 5\% dextrose solutions associated with higher rates of adverse events than intravenous $5 \%$ dextrose solutions?" and "In elderly, patients is rehydration with subcutaneous $5 \%$ dextrose solutions as effective as intravenous $5 \%$ dextrose solutions?"

\section{Methods}

\section{Data sources}

We carried out a comprehensive search of the literature including the Cochrane Library, Medline, IDIS, CINAHL, Current Contents, Premedline, Australasian Medical Index, the Joanna Briggs Institute and the United States National Guideline Clearinghouse (Table 1). Due to the cost of access, Embase was not searched directly, however the Cochrane Central Register of Controlled Trials (part of the Cochrane Library) was searched and it includes details of controlled trials from Embase.

Our search strategy combined terms for subcutaneous fluid infusion (hypodermoclysis, clysis, fluid therapy, infusion, subcutaneous injection) with terms for the administered solution (dextrose, glucose), and terms to restrict the search to elderly participants (aged) (Table 3 ).

\section{Study selection and data extraction}

The clinician requested that we only include articles published in English in the last 10 years as it was felt that articles published prior to this might not reflect current clinical methods.

We identified a relevant systematic review published in 1997. As a result, we only appraised the systematic review and relevant articles published after (or not included in) the systematic review.

From our sources we identified 15 potentially relevant articles. We obtained the full text of these articles to determine their relevance. Eleven of the articles were excluded on the basis that they were either narrative review or editorial $(n=6)$, evaluated in the included systematic review $(\mathrm{n}=2)$, undertaken in an inappropriate patient group ( $\mathrm{n}$ $=2)$ or were case series or case reports $(n=1)$.

The systematic review, two randomised controlled trials (RCT) and one cohort study then remained and were critically appraised.

\section{Results and discussion}

The systematic review by Rochon et al [2] aimed to evaluate the evidence supporting the use of hypodermoclysis to treat dehydration in elderly patients. The review evaluated efficacy and safety data on hypodermoclysis from 13 studies, which investigated the effects of a range of different administered fluids, including some dextrose solutions.

The review included a limited search strategy involving only Medline and reference lists, and provided detailed inclusion/exclusion criteria. To be included in the review studies had be published in English, contain original patient data and address the infusion of fluids to treat dehydration in adults. Articles reporting infusion of 
Table 2: Details of Included Studies

\begin{tabular}{|c|c|c|c|c|}
\hline Study & No of Patients & Type of Study & Conclusions & Appraiser's Comments \\
\hline Rochon et al 1997 [2] & I3 studies included & Systematic Review & $\begin{array}{l}\text { Hypodermoclysis of } 40 \mathrm{~g} / \mathrm{L} \text { dextrose } \\
\text { and } 30 \mathrm{mmol} / \mathrm{L} \mathrm{NaCl} \text { or } 5 \% \text { dextrose } \\
\text { solution and } 4 \mathrm{~g} / \mathrm{L} \mathrm{NaCl} \text { is safe and } \\
\text { effective }\end{array}$ & $\begin{array}{l}\text { Limited search strategy. Minimal } \\
\text { appraisal of validity of included studies. }\end{array}$ \\
\hline Slesak et al 2003 [3] & 96 & Randomised Controlled Trial & $\begin{array}{l}\text { Hypodermoclysis of half-normal } \\
\text { saline-glucose } 5 \% \text { is well accepted by } \\
\text { elderly patients and is of comparable } \\
\text { efficacy and safety to IV rehydration. }\end{array}$ & $\begin{array}{l}\text { Large number of switches between } \\
\text { treatment groups and used non- } \\
\text { validated measures of discomfort and } \\
\text { feasibility. }\end{array}$ \\
\hline O'Keeffe \& Lavan 1996 [4] & 60 & Randomised Controlled Trial & $\begin{array}{l}\text { SC or IV infusion have comparable } \\
\text { efficacy and safety. SC infusion is } \\
\text { better tolerated by elderly confused } \\
\text { patients }\end{array}$ & $\begin{array}{l}\text { Data on dextrose solutions not } \\
\text { reported separately. No blinding. }\end{array}$ \\
\hline Dasgupta, Binns \& Rochon 2000[5] & 55 & Cohort Study & $\begin{array}{l}\text { Hypodermoclysis with two-thirds } 5 \% \\
\text { glucose and one-third normal saline or } \\
\text { normal saline was as effective as IV } \\
\text { infusion and associated with local } \\
\text { reactions and complications. }\end{array}$ & $\begin{array}{l}\text { Substantial methodological } \\
\text { weaknesses. Allocation to treatment } \\
\text { based on physician preference, small } \\
\text { sample size in IV group, non-validated } \\
\text { outcome measures. }\end{array}$ \\
\hline
\end{tabular}

medications or contrast media were excluded, as were articles that focussed on sensitivity to hyaluronidase.

The review had a number of limitations including the restricted search strategy and minimal assessment of validity of included trials. Little analysis or synthesis of results was presented and no statistical meta-analysis was undertaken. Studies were simply grouped and discussed according to study design.

The evidence appraised in the systematic review [2] did not demonstrate a significant difference in absorption or adverse events when $40 \mathrm{~g} / \mathrm{L}$ dextrose with $30 \mathrm{mmol} / \mathrm{L}$ $\mathrm{NaCl}$ or $5 \%$ dextrose solution with $4 \mathrm{~g} / \mathrm{L} \mathrm{NaCl}$ was delivered via SC or IV routes.

The authors did not make any specific conclusions regarding dextrose solutions but concluded that hypodermoclysis could be used effectively for the treatment of dehydration. They also noted that given that dehydration is so common in the elderly, and hypodermoclysis is viable in long-term care settings, it was important that a definitive investigation of hyperdermoclysis be undertaken. [2].

The second study, by Slesak et al [3], was a randomised controlled trial including 96 elderly patients (mean age 85.3 years) in German hospital geriatric wards with signs of mild to moderate dehydration needing parenteral fluids, over a period of 20 months. Patients with infectious and allergic skin diseases, generalised oedema, acute myocardial infarction, or other medical conditions in which the study regime would be inappropriate were excluded. Those known to be intolerant to one of the methods of infusion, in manifest or imminent shock, requiring intravenous drug administration or total parenteral nutrition, participating in another study or who refused consent were also excluded.

Participants were block randomised to either subcutaneous (SC) infusion of half-normal saline-glucose $5 \%$ or intravenous (IV) infusion of half-normal saline-glucose $5 \%$. The groups had similar baseline characteristics. Blinding of patients, clinicians or assessors was not attempted.

Data collected included nurses' and doctors' scores of feasibility of the procedure, patient ratings of discomfort, occurrence of complications and length of hospital stay. A number of measures were also assessed before and after therapy including blood pressure, heart rate, haematocrit, sodium, creatinine, activities of daily living (ADL) and orientation to person, place and time.

A large percentage of the participants did not remain in the treatment group to which they were allocated. Patients allocated to SC infusion changed to IV in 13 cases $(27 \%)$, 11 times because of the need to administer drugs by IV and twice as a result of poor absorption. Patients allocated to IV infusion changed to SC infusion in 17 cases (35\%). Eight changes were due to impossibility of continuous peripheral venous puncture, five were due to permanent removal of cannula by the patient, the remaining four were made for miscellaneous reasons. An intention-totreat analysis was undertaken. As a result of the significant movement between treatment groups (in both directions), the results of four groups (IV only, IV/SC, SC only, SC/IV) were also compared.

The degree of movement between treatment groups in this study introduces substantial opportunity for bias. Characteristics of the patients who changed groups were not provided, so it is difficult to assess whether they were representative of the wider population or a particular sub- 
Table 3: Search Strategy for Medline (Similar terms were used in other databases)

\begin{tabular}{ll}
\hline 1 & (Hypodermoclysis or clysis).mp \\
2 & Fluid therapy/or rehydration.mp \\
3 & Exp Infusions, parenteral/ \\
4 & Exp Injections, subcutaneous/ \\
5 & Dehydration/ \\
6 & or/l-5 \\
7 & Exp Glucose/or glucose.mp \\
8 & Dextrose.mp \\
9 & 7 or 8 \\
10 & Exp Aged/or elderly.mp \\
11 & 6 and 9 and I0 \\
12 & Limit II to (human and English language and yr $=1993-2003)$ \\
\hline
\end{tabular}

group. Results were presented as medians with range or interquartile range. The study employed measures of feasibility and discomfort that were not validated which weakens the validity of the conclusions.

In the RCT conducted by Slesak et al [3], median length of hospital stay and duration of fluid infusion did not vary significantly between patients having IV and SC infusions, however the median (minimum; maximum) volume of IV infusions was significantly greater than SC infusions $(1000(500 ; 1500) \mathrm{mL} / \mathrm{d}, 750(457 ; 1500) \mathrm{mL} / \mathrm{d}, \mathrm{p}=$ $0.002)$. Using intention-to-treat analysis, there was no difference in patient discomfort or nurses' feasibility score between the two treatment groups. Doctors rated SC infusion as significantly more feasible than IV infusion (SC: median = $2($ IQR:1.25-2), IV: 2(2-3.875), p = 0.011). Clinical and laboratory measures were not significantly different between groups and there were no significant differences between type or frequency of side effects in the two groups.

The authors concluded that rehydration by SC and IV infusion were equally well accepted by geriatric patients, similarly feasible and comparably safe and effective. They also noted the usefulness of SC infusion in confused patients and in those in whom IV punctures are difficult to achieve. [3].

O'Keeffe and Lavan [4] also carried out a RCT of subcutaneous fluids in elderly hospital patients in Britain. In this study the 60 participants (mean age 80 years) were cognitively impaired patients admitted to an acute geriatric unit who required parenteral fluids for at least 48 hours because of mild dehydration on poor oral intake. Patients were excluded if they required IV medications, if more than 2 litres of fluid was required in a 24 hour period, if there was clinical evidence of poor tissue perfusion or if the precise amount of fluid administered was judged to be critical (ie as a result of renal or heart failure).
The participants were randomly allocated to SC infusion or IV infusion. In both treatment groups, patients received either $0.9 \%$ saline, $0.45 \%$ saline or $5 \%$ dextrose. The two groups had similar baseline characteristics. The amount of fluid prescribed, amount of fluid administered, patient agitation, number of cannula required, serum urea and creatinine were measured.

Blinding was not undertaken. Participants were randomised to treatment groups in blocks of six. A sealed envelope containing the treatment allocation was opened after a patient had been entered into the study. One patient switched from IV to SC fluids due to difficulty gaining venous access and one patient in the SC group died for reasons unrelated to the infusion. These two patients were excluded from the analysis. Data was provided for a mixed sample of infusion fluids, data on dextrose administration was not reported separately.

In contrast with Slesak et al [3], the RCT by O'Keeffe and Lavan [4] found no significant difference in the amount of fluid or the proportion of prescribed mean (standard deviation) volume administered by IV as compared to SC infusion $(0.76(0.14)$ litres vs $0.82(0.12)$ litres, $\mathrm{p}=0.1)$. There was also no significant difference found between serum creatinine or urea between the two groups. Agitation relating to the drip or cannula was noted in 11 (37\%) of the patients on SC fluids and $24(80 \%)$ of the patients on IV fluids (RR $0.46,95 \% \mathrm{CI}(0.28-0.76), \mathrm{p}=0.0007$ ). Re-siting of the infusion was required in four (13\%) of the SC group and seven $(23 \%)$ of the IV group, however this difference was non-significant (RR 0.57, 95\%CI(0.19 $1.75), p=0.32$ ). Local oedema was noted in two patients receiving subcutaneous fluids, no other complications were noted.

The authors concluded that study demonstrated the advantages of SC infusion in elderly, cognitively impaired patients with relatively mild dehydration. They noted that 
patients were less likely to attempt to interfere with SC infusions.

The fourth study identified (Dasgupta, Binns and Rochon [5]) was a cohort study of subcutaneous fluid infusion in a long-term care setting in Toronto, Canada. In this observational study, 55 residents of a long-term care facility were treated with fluid therapy during a five week period. To be included, patients had to receive either SC or IV fluids during the five week study period. Patients with only one eight-hour shift of fluid therapy, and those receiving medications through hypodermoclysis were excluded, as were those who received blood products and those with life-threatening conditions.

Thirty-seven of the patients received hypodermoclysis, nine received IV fluids, and nine received both. Fluids infused by hypodermoclysis were two-thirds $5 \%$ glucose with one-third normal saline, or normal saline. Intravenous fluid therapy consisted of administration of twothirds $5 \%$ glucose with one-third normal saline, half-normal saline or normal saline. No blinding was undertaken and allocation to treatment group was dependent on physician preference. The treatment groups differed in size and only limited data on baseline characteristics of the groups were provided.

The authors measured occurrence and type of adverse effects, clinical or laboratory evidence of hydration improvement, including correction of hypernatremia, or reduction in the $\mathrm{BUN} /$ creatinine ratio to $<25$.

This study had a number of substantial methodological weaknesses. Allocation to treatment based on physician preference introduces considerable opportunity for bias and this is increased by the limited analysis of baseline differences between treatment groups. The small sample size, particularly in the IV group ( $n=9)$, is another weakness of this study. All patients having chronic treatment received SC fluids. The lack of comparison with fluid therapy by IV in patients in this group limits the value of data from this part of the study. Additionally, pre-treatment laboratory evidence of dehydration was only present in five $(18 \%)$ patients on hypodermoclysis for acute treatment introducing a ceiling effect that limits the ability of the study to observe an improvement in hydration status. Use of non-validated measures of improvement ("general" and "clinical" improvement) further limits validity of results, as does the reporting of data including a variety of administered fluids.

Similarly to the article by O'Keeffe and Lavan [4], the results of the cohort study [5] showed no significant differences between the IV and SC groups for general improvement, clinical improvement or laboratory improvement. Patients receiving hypodermoclysis had a significantly lower mean number of complications per day (SD) $(0.07(0.16), 0.21(0.25), \mathrm{p}=0.04)$ and a significantly lower mean $( \pm S D)$ number of local reactions per day of therapy $(0.05(0.10), 0.20(0.25), \mathrm{p}=0.02)$. There were no significant differences between the groups in regard to the mean number of attempts to remove the catheter or mean number of possible fluid overload episodes per day of therapy.

Dasgupta, Binns and Rochon [5] concluded that SC infusion was a safe alternative to IV fluids treatment for elderly residents in a long-term care setting with mild to moderate dehydration.

\section{Conclusions}

The four reviewed studies, including one systematic review and two randomised-controlled trials, as well as one less methodologically rigorous study, all provide evidence that appropriate volumes of subcutaneous dextrose infusions (in the form of half-normal saline-glucose $5 \%$, $40 \mathrm{~g} / \mathrm{L}$ dextrose and $30 \mathrm{mmol} / \mathrm{L} \mathrm{NaCl}$, or $5 \%$ dextrose solution and $4 \mathrm{~g} / \mathrm{L} \mathrm{NaCl}$, or two-thirds $5 \%$ glucose and one-third normal saline) can be used effectively for the treatment of dehydration in elderly patients, with similar rates of adverse effects to intravenous infusion.

However, the evidence in this area is limited and the studies appraised each have methodological flaws that limit the strength of the conclusions that can be drawn. Larger, methodologically rigorous, randomised controlled trials using validated outcome measures would be useful to confirm these results.

\section{Competing interests}

None declared.

\section{Authors' contributions}

TT carried out the literature search and critical appraisal of included articles and drafted the article. AC developed the research question and provided clinical expertise as well as providing comments on the draft. Both authors read and approved the final manuscript.

\section{References}

I. Dangers of hypodermoclysis. Nutr Rev 1953, I I:232-234.

2. Rochon PA, Gill SS, Litner J, Fischbach M, Goodison AJ, Gordon M: A systematic review of the evidence for hypodermoclysis to treat dehydration in older people. J Gerontol A Biol Sci Med Sci 1997, 52:M169-176.

3. Slesak G, Schnurle JW, Kinzel E, Jakob J, Dietz K: Comparison of subcutaneous and intravenous rehydration in geriatric patients: a randomized trial. J Am Geriatr Soc 2003, 5 I: I55-I60.

4. O'Keeffe ST, Lavan JN: Subcutaneous fluids in elderly hospital patients with cognitive impairment. Gerontology 1996, 42:36-39.

5. Dasgupta M, Binns MA, Rochon PA: Subcutaneous fluid infusion in a long-term care setting. J Am Geriatr Soc 2000, 48:795-799. 


\section{Pre-publication history}

The pre-publication history for this paper can be accessed here:

http://www.biomedcentral.com/1471-2318/4/2/prepub

Publish with Bio Med Central and every scientist can read your work free of charge

"BioMed Central will be the most significant development for disseminating the results of biomedical research in our lifetime. " Sir Paul Nurse, Cancer Research UK

Your research papers will be:

- available free of charge to the entire biomedical community

- peer reviewed and published immediately upon acceptance

- cited in PubMed and archived on PubMed Central

- yours - you keep the copyright 